\title{
Aberration-corrected STEM of Four-atom Rhenium Nanowires Confined within Carbon Nanotubes
}

\author{
Fan Zhang ${ }^{1}$, Pengju Ren ${ }^{1}$, Xiulian Pan ${ }^{1}$, Xinhe Bao ${ }^{1}$ and Jingyue Liu ${ }^{2}$ \\ 1. State Key Laboratory of Catalysis, Dalian Institute of Chemical Physics, Chinese Academy of \\ Sciences, Dalian 116023, China. \\ 2. Department of Physics, Arizona State University, Tempe, Arizona 85287, USA.
}

Due to the spatial confinement effect nanowires (NWs) of just a few atoms thick in diameter can possess novel quantum properties. These ultrathin NWs, however, tend to be both chemically and structurally unstable. Carbon nanotubes (CNTs), on the other hand, can be used as templates to synthesize nanostructures that remain stable [1-5]. For example, nanofilling of Eu can produce single-atom chains in double-wall CNTs (DW-CNTs) [3]. Self-assemble of graphene nanoribbons within single-wall CNTs and the helical twist and screw-like motion of the carbon nanoribbons have been observed [4]. Monocrystalline FeCo NWs inside CNTs with unique magnetic properties have been synthesized [5]. The strong metal-CNT interaction and the confinement effects of small diameter CNTs can induce the formation of novel metal phases that are generally not stable. We report here the discovery of selfassembled, ultra-long and atomically thin Re NWs with an unusual fcc-stacking pattern along the length of the CNTs. Re usually possesses a hcp structure which is extremely stable and no phase transition occurs under pressures to $216 \mathrm{GPa}$ and temperatures up to its melting point [6].

Purified DW-CNTs with inner diameters $<1.5 \mathrm{~nm}$ were selected as templates to grow Re NWs. Volatile methyltrioxorhenium $\left(\mathrm{Re}\left(\mathrm{CH}_{3}\right) \mathrm{O}_{3}\right)$ was used as the precursor material to fill in the CNT channels. After being washed, dried, reduced in $\mathrm{H}_{2}$ for $2 \mathrm{~h}$ at $823 \mathrm{~K}$, and annealed in He for $12 \mathrm{~h}$ at $473 \mathrm{~K}$, the encapsulated organometallic Re species transformed into clusters dispersed within the CNT channels. Upon repeating the above annealing cycles for 3 times, the discrete Re nanoclusters self-assembled into continuous NWs within the CNT channels. Repeated annealing cycles produced longer Re NWs. Aberration-corrected STEM (AC-STEM), especially high-angle annular dark-field (AC-HAADF), technique was used to image the atomic arrangement of the ultrathin Re NWs within the CNT channels.

Figure 1 shows a typical atomic resolution HAADF image of a Re nanowire, self-assembled within DWCNTs. The bright dots represent Re atoms. Some individual Re atoms (indicated by the white arrow in Fig. 1a), were also observed. By using the intensity of the individual Re atoms as an internal standard, one can deduce that the brighter dots in the center of the Re NW (Fig. 1a) contains two overlapping Re atoms and the dim dots on each side of the central brighter line of dots contain only one $\mathrm{Re}$ atom. Detailed analysis of many similar Re NWs (e.g., Fig 1b and 1c) suggests that the stacking of the Re atoms follows closely to that of the fcc Re projected along the [110] zone axis with the [1-10] direction along the length of the CNT. Figure 1c shows an interesting Re NW with different arrangement of the Re atoms along the CNT length. By comparing to the projections of a four-atom fcc Re NW (oriented with the [1-10] direction along the CNT length), it was identified that the different segments of the re NW shown in Fig. 1c correspond to the four-atom fcc Re NW projected along the [110] (indicated by the solid block arrow), the [111] (indicated by the hollow block arrow), and the [112] (indicated by the red arrow) zone axis, respectively. It is not clear why the Re NW existed in different configurations within the CNT. Stacking defects are, however, observable along the ultrathin Re NWs. DFT calculations revealed that the strong interaction between the $\mathrm{Re}$ atoms and the $\mathrm{C}$ atoms of the CNTs 
played a vital role in determining the formation of the fcc Re NW. Figure 2a shows the optimized model of Re4@ $(7,7)$. The calculation of the lattice parameters suggests that the encapsulated Re fcc structure is distorted, i.e. compressed along [001] and stretched along [1-10], which implies an extremely strong interaction between the $\mathrm{Re}$ atoms and the $\mathrm{C}$ atoms of the CNT walls. Figure $2 \mathrm{~b}$ and $2 \mathrm{c}$ shows, respectively, the electron density around the vicinity of the different $\mathrm{Re}$ atoms. The \#1 and \#3 Re atoms donate electrons to the $\mathrm{C}$ atoms and the other two Re atoms \#2 and \#4 do not show obvious electron transfer. This uneven interaction leads to a distorted Re fcc structure and causes deformation of the CNTs into a slight oval shape when viewed along its length. The inner spaces of CNTs can be used as nanoscale reactors to synthesize novel structures with unique properties.

\section{References:}

[1] J. Lee et al, Nature 415 (2002), p.1005.

[2] L.-J. Li et al, Nature Materials 4 (2005), p. 481.

[3] R Kitaura et al, Angew. Chem. Int. Ed. 48 (2009), p. 8298.

[4] A. Chuvilin et al, Nature Materials 10 (2011), p. 687.

[5] A. L. Elías et al, Nano Lett. 5 (2005), p. 467.

[6] Y. K. Vohra et al, Phys Rev B 36 (1987), p. 9790.

[7] The authors acknowledge the use of facilities in the John M. Cowley Center for High Resolution Electron Microscopy at Arizona State University.
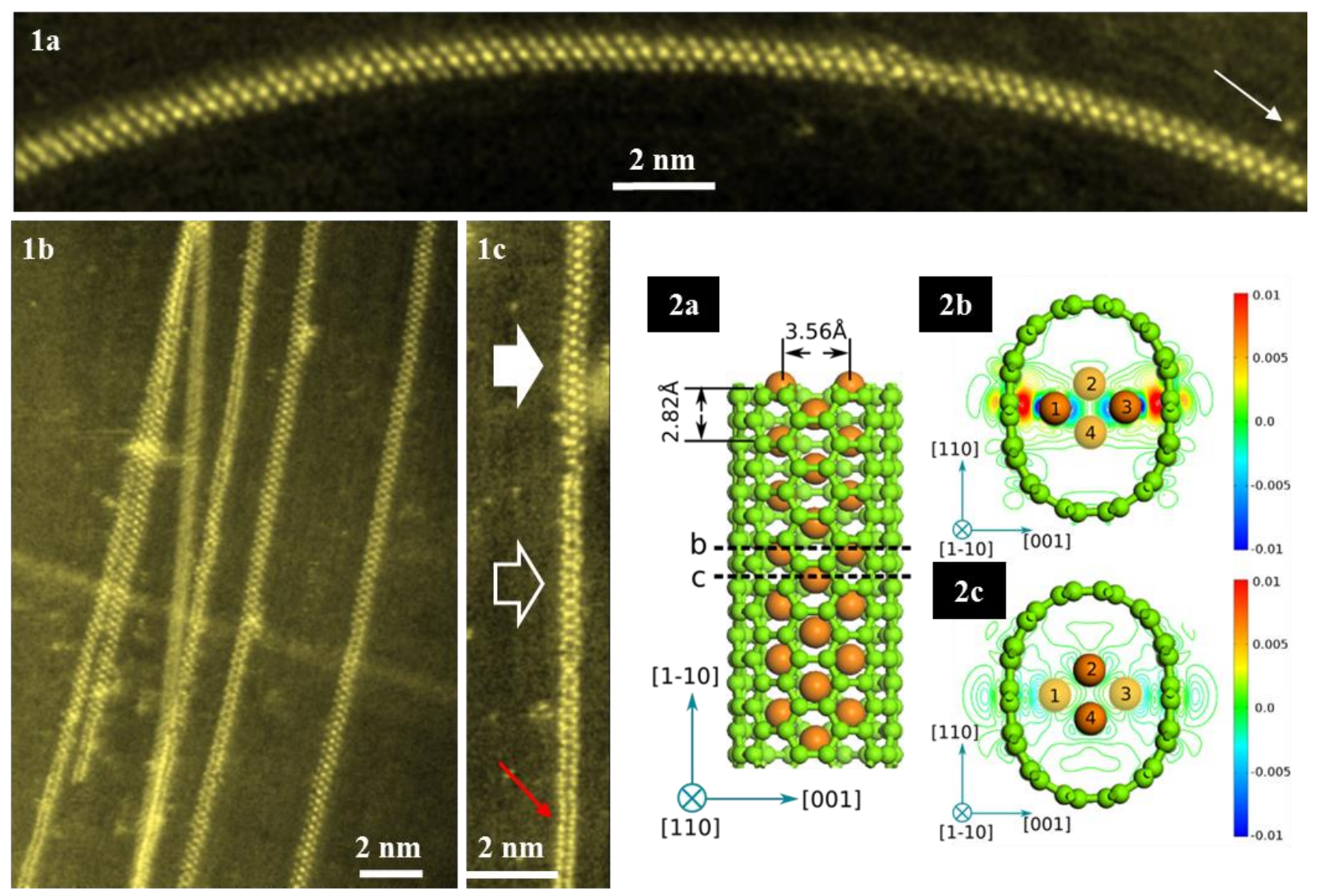

Figure 1. HAADF images of self-assembled, four-atom Re NWs within double-wall carbon nanotubes. Figure 2. DFT calculations of the interaction of the four-atom fcc Re NW with the carbon atoms of the double-wall CNT. The side scale bars indicate the degree of electron transfer from Re to C. 